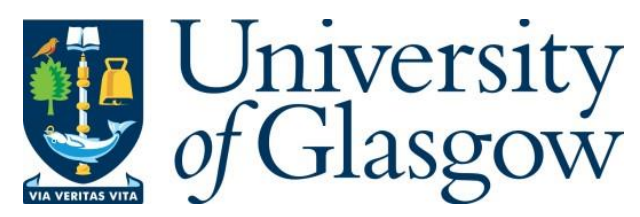

Feeney, A. and Lucas, M. (2018) A comparison of two configurations for a dualresonance cymbal transducer. IEEE Transactions on Ultrasonics, Ferroelectrics, and Frequency Control, (doi:10.1109/TUFFC.2018.2793310).

There may be differences between this version and the published version. You are advised to consult the publisher's version if you wish to cite from it.

http://eprints.gla.ac.uk/144451/

Deposited on: 11 January 2018

Enlighten - Research publications by members of the University of Glasgow http://eprints.gla.ac.uk 


\title{
A Comparison of Two Configurations for a Dual-Resonance Cymbal Transducer
}

\author{
Andrew Feeney and Margaret Lucas (Corresponding Author)
}

\begin{abstract}
The ability to design tuned ultrasonic devices that can be operated in the same mode at two different frequencies has the potential to benefit a range of applications, such as surgical cutting procedures where the penetration through soft then hard tissues could be enhanced by switching the operating frequency. The cymbal transducer has recently been adapted to form a prototype ultrasonic surgical cutting device that operates at a single frequency. In this paper, two different methods of configuring a dual-resonance cymbal transducer are detailed. The first approach relies on transducer fabrication using different metals for the two end-caps, thereby forming a dual-resonance transducer. The second employs transducer end-caps composed from a shape memory alloy, superelastic Nitinol. The resonance frequency of the Nitinol transducer depends on the phase microstructure of the material, switchable through the temperature and/or stress dependency of the Nitinol end-caps. The vibration response of each transducer is measured through electrical impedance measurements and laser Doppler vibrometry, and finite element analysis is used to show the sensitivity of transducer modal response to the fabrication processes. Through this research, two viable dual-resonance cymbal transducers are designed and characterised, and compared to illustrate the advantages and disadvantages of the two different approaches.
\end{abstract}

Index Terms-Frequency measurement and statistics, Transducer Modeling (FEA and Analytical), Transducer Fabrication Technology

\section{INTRODUCTION}

$\mathrm{T}$ HE The ability to change the resonance frequency or the operating resonance mode of an ultrasonic device is beneficial in a range of applications. Examples include devices for energy harvesting [1] where the adjustment of resonance frequency of the harvester is necessary to adapt to different environmental vibration conditions, and in cavitation generation [2] where multi-frequency sonication at ultrasonic frequencies has been reported to increase cavitation yield. A tuneable ultrasonic device for manufacturing processes has been reported for mechanical turning [3] where the ultrasonic vibrations reduce tool-wear and the opportunity to drive the device at different resonance frequencies means that switching tools for different operations can be avoided.

In many ultrasonically-assisted processes, the ultrasonic device is required to operate on or within several different

A. Feeney was with the School of Engineering, University of Glasgow, Glasgow G12 8QQ, UK. He is now with the Department of Physics, University of Warwick, Coventry CV4 7AL, UK (e-mail: a.feeney@warwick.ac.uk). media, presenting different loads to the device throughout the process. Example applications include ultrasonic cutting of multi-layered food products and ultrasonic surgical devices that are required to penetrate both soft and hard tissues. As a consequence, there are many applications where it would be advantageous to be able to select the ultrasonic response of the device, possibly including frequency, operating mode shape and ultrasonic amplitude, to optimally interact with each material or medium involved in the process.

\section{A. The Cymbal Transducer}

The cymbal transducer is a flextensional-type configuration, developed as a modification of the moonie design [4], and has principally been utilised in underwater applications such as in hydrophone arrays for both sensing and projection [5]. Recent research has demonstrated the potential of the cymbal for power ultrasonics applications [6-8] and a modification of the classical cymbal design, with a single end-cap, has been developed as a prototype ultrasonic orthopaedic surgical device [9].

The cymbal transducer comprises a piezoelectric element, in either a disc or ring configuration, coupled to two cymbalshaped end-caps, typically bonded using an epoxy resin. A radial motion of the piezoelectric element is generated through an input voltage, which is converted into a comparatively high axial displacement end-cap vibration, achieving an amplification of the order of 40 [4]. It is known that the majority of cymbal transducers exhibit a dual-resonance response [8,1012] as an undesirable consequence of the end-cap fabrication and transducer assembly processes. Often a double-resonance exhibits in the frequency response due to small asymmetries, as a result of even minor differences in the dimensions of the two end-caps or an uneven epoxy resin bond layer. The two resonance modes are characterised by out-of-phase and inphase vibration of the end-caps, which are the symmetric and asymmetric modes of vibration respectively. Although tolerances of end-cap dimensions can be controlled, it is more difficult to regulate the physical dimensions of the epoxy layer during the curing process. The frequencies of a dual-resonance caused by slight asymmetries tend to be in close proximity, often only a few tens or hundreds of $\mathrm{Hz}$ apart, but these inadvertent dual-resonances show that there is potential for designing a dual-resonance cymbal transducer by controlling the differences in the dimensions and mechanical properties of

M. Lucas is with the School of Engineering, University of Glasgow, Glasgow G12 8QQ, UK. (e-mail: margaret.lucas@glasgow.ac.uk). 
the components of the device. A significant advance in the design of a selectable resonance frequency transducer was based on a modification of the cymbal transducer [13], where the two cymbal end-caps were either made from different metals or made with different geometries. For both design solutions, the transducer could be operated at two different resonance frequencies.

A general advantage of the cymbal transducer is that, unlike ultrasonic devices based on a Langevin transducer, an attached tool does not have to be tuned to the resonance frequency of the transducer. This allows considerably more freedom in the design of the attachment, which can focus on meeting the needs of the tool/material interaction rather on a geometry that is tuned. The cymbal is designed initially with a resonance frequency well above the desired operating frequency and then the added mass of the attached tool brings the resonance frequency down and results in a device that is resonant at the required operating frequency. As a consequence, another modification of the classical cymbal design has recently been demonstrated as the transducer of a prototype ultrasonic orthopaedic surgical device [9], where the added mass is a cutting tip. This transducer now offers an opportunity for incorporating a capability for operating in a selectable resonance condition. The capacity to select from two operating resonance frequencies of the transducer, for the same operating mode, would enable the cutting device to be more optimised for the properties of the material being cut. In surgical applications, as well as improving cutting efficiency, this could bring additional benefits such as allowing for more surgical procedures to be carried out with the same cutting tip. One possible design solution is to incorporate a phase transformation shape memory alloy (SMA) in the transducer and this approach has been investigated in recent research, where the SMA Nitinol was used in cymbal transducers in order to generate a tuneable resonance capability through phase transformation of the material [14-16].

\section{B. Nitinol for Tuneable Resonance Devices}

The mechanical properties of Nitinol alter in response to changes in temperature or stress $[17,18]$, where the material transforms between different microstructural states, exhibiting shape memory and superelasticity. Austenitic Nitinol forms at a temperature which depends on the alloy composition, and is a stiff cubic B2 phase, whereas martensitic Nitinol forms at a comparatively lower temperature, and is a soft B19' phase $[17,19]$. Superelasticity is a material phenomenon associated with Nitinol which is principally stress-induced [20]. Once Nitinol has completely transformed to austenite, the application of a mechanical load can reorient the microstructure to the softer martensite phase, thereby generating the superelastic effect in the Nitinol. Upon unloading, this transformation to martensite instantaneously reverses. In the study of Nitinol transducers, the influence of the superelastic effect has thus far been largely neglected. The properties of Nitinol are influenced by the chemical composition of the alloy. Nitinol can therefore be tailored to exhibit the superelastic effect at a particular temperature. For superelastic Nitinol, the phase transition to austenite commonly occurs below room temperature. Devices which currently exploit the superelastic effect include biomedical stents [21]. A Nitinol stent is compressed in its superelastic state, where the microstructure phase reorients from austenite to martensite, before insertion into a patient. Once the compressive load is removed, the material microstructure forms austenite. The Nitinol must be in a superelastic state for the stent to operate properly, and the bodytemperature of the patient commonly provides the essential environmental conditions.

However, despite some successes in its use in devices, the relatively poor understanding of how phase transformations affect the mechanical properties of Nitinol is a current restriction on the application of the material [22]. The mechanical properties and the transformation temperatures of Nitinol are sensitive to stress, meaning that, for adoption in cymbal transducers, the end-cap fabrication process must be controlled to avoid an undesired shift of the transformation temperatures. Mechanical and thermal processing of Nitinol should be used with caution, since heat treatment and cold working both affect the transformation temperatures [19,23]. Nitinol can exhibit secondary deformation during mechanical testing, such as stress-induced martensite, and this has contributed to problems in being able to measure its mechanical properties [22]. Therefore, using conventional mechanical testing methods to measure the material properties of Nitinol, such as elastic modulus, have often not been successful [24].

\section{Challenges for Tuneable Resonance Transducers}

The resonance frequency of a cymbal transducer is very sensitive to small changes in the end-cap dimensions. Furthermore, the fabrication of cymbal end-caps from Nitinol is complicated by the difficulty in controlling the mechanical and phase transformation properties. Although Nitinol has thus far exhibited promise as a candidate material for the manufacture of tuneable resonance devices [14-16], the vibration response and characteristics of a cymbal transducer fabricated with superelastic Nitinol requires investigation if practical applications are to be realised.

The main challenges in the design and fabrication of tuneable resonance cymbal transducers are addressed here by comparing two different transducer design approaches. The first is a cymbal transducer called a binary transducer, where the two end-caps are made of metals with different Young's modulus, enabling two selectable resonance operation frequencies and modes. The second cymbal transducer is a Nitinol transducer, where the end-caps are fabricated from superelastic Nitinol.

\section{METHODOLOGY}

The basis of this study is to demonstrate two different ways in which cymbal transducers can be configured to enable switching between two resonance frequencies. The cymbal transducer has been selected based on its fundamental simplicity in design and manufacture. Furthermore, the configuration is ideal for the generation of a dual-resonance frequency response, where end-caps of differing physical properties can be attached. 
Finite element analysis (FEA, Abaqus/CAE, Dassault Systèmes) is used for the design of end-caps, and to model, characterise and tune the resonance frequencies and mode shapes of the binary and Nitinol transducers. For the binary transducer, FEA is used to design a transducer which results in two distinct and well-separated resonance frequencies of the fundamental modes of vibration. The two different metals chosen for the end-caps are silver steel and titanium, which have suitably different Young's modulus. The design of the Nitinol transducer is more complicated, since the elastic modulus of the Nitinol end-cap is very sensitive to temperature and the end-cap fabrication process. The Nitinol material itself comes with supplier estimations of properties associated with the material composition, but these are not accurately representative of the properties of the fabricated end-cap. However, FEA was used to design the cymbal transducer and estimate suitable end-cap dimensions, based on estimated properties, but was not used to tune or characterise the cymbal device at the design stage.

The fabricated transducers were initially characterised by measuring the impedance-frequency response using electrical impedance analysis (Agilent 4294A Impedance Gain/Phase Analyzer). The binary transducer was characterised using this method at room temperature, but the characterisation approach for the Nitinol transducer was adapted. Nitinol is superelastic up to approximately $50^{\circ} \mathrm{C}$ above the final austenitic transformation temperature, $A_{F}$ [25]. The measurement of $A_{F}$ has been previously reported [16], with the transition to complete austenite in the Nitinol end-caps (Johnson Matthey Noble Metals), providing an indicator of $\mathrm{A}_{\mathrm{F}}$, measured as approximately $45^{\circ} \mathrm{C}$. The same superelastic Nitinol was used to fabricate the end-caps for the Nitinol transducer, and so a minimum temperature limit of $45^{\circ} \mathrm{C}$ was set in the characterisation process to generate the austenite phase, and hence ensure the required conditions for superelasticity. In each case where temperature control is referenced, an experimental thermally controlled chamber is used and the temperature is monitored using a type-K thermocouple and an infrared thermal imaging camera (FLIR T425). In the case of impedancefrequency response measurements for the Nitinol transducer, characterisation through a temperature range enabled the temperature-modulus dependence of the Nitinol end-caps to be demonstrated.

Experimental modal analysis (EMA) of each transducer is performed to extract the resonance frequencies and associated mode shapes. The EMA is carried out at room temperature using a 3D laser Doppler vibrometer (Polytec CLV) to record the displacement-frequency response from a grid of measurement locations on the cymbals. The frequency response functions are post-processed in EMA software (ME'Scope) to provide a wire-frame animation of the cymbal mode shapes.

The final stage of the characterisation process is the analysis of transducer response when driven through resonance. Harmonic analysis uses a 1D laser Doppler vibrometer (Polytec CLV) to measure the displacement amplitude of vibration across a frequency range through resonance, measuring from the centre of whichever of the two cymbal end-caps dominates the displacement response. The transducers are excited by a burst-sine signal, to avoid the effects of heating, with an excitation voltage of $2 \mathrm{~V}$, controlled through a LabVIEW interface. The amplitude-frequency response of both modes of the binary transducer is measured.

The harmonic analysis procedure is adapted for the Nitinol transducer because there is no understanding of the influence of superelasticity on the vibration response. First, the temperature of the Nitinol transducer is increased to ensure the end-caps have completely transformed to austenitic Nitinol, then the displacement response through resonance at an excitation voltage of $2 \mathrm{~V}$ is measured. Subsequently, the temperature is maintained high enough to ensure the Nitinol end-caps are in the austenitic phase. The excitation voltage is then increased from $20-220 \mathrm{~V}$, in $20 \mathrm{~V}$ increments. As the displacement amplitude of the end-cap increases with excitation voltage, the superelastic effect will cause local transformations to martensite. This experiment aims therefore to capture how this impacts on the measured response of the transducer.

\section{FABRICATED TRANSDUCERS}

The dimensions of end-caps directly affect the resonance characteristics of the transducer [26]. In this case, the end-caps of the binary transducer were milled. The manufacture of endcaps is generally challenging to control, and it has been reported that only around $20 \%$ of all fabricated cymbal transducers exhibit a single-peak response in the frequency spectrum that indicates a symmetric device, with tight end-cap and bond layer tolerances [12]. The transducer resonance frequency is primarily dependent on the dimensions and elastic properties of the end-caps, with the base cavity diameter and the end-cap thickness particularly influencing the vibration response [26]. The dimensions of end-caps for the binary and Nitinol transducers are shown in Table I.

TABLE I

END-CAP DIMENSIONS IN MILLIMETRES

\begin{tabular}{cccc}
\hline \hline Dimension & Silver Steel & Titanium & $\begin{array}{c}\text { Superelastic } \\
\text { Nitinol }\end{array}$ \\
\hline Apex Cavity Diameter & 4.56 & 4.25 & 4.50 \\
Base Cavity Diameter & 9.74 & 9.55 & 9.10 \\
Cavity Depth & 0.31 & 0.30 & 0.31 \\
End-Cap Thickness & 0.21 & 0.22 & 0.24 \\
Total Diameter & 12.71 & 12.71 & 12.72 \\
End-Cap Height & 0.52 & 0.52 & 0.55 \\
\hline \hline
\end{tabular}

The binary transducer end-caps are fabricated from silver steel rod (elastic modulus $200 \mathrm{GPa}$ [27]) and titanium Ti-6Al$4 \mathrm{~V}$ sheet (elastic modulus $110 \mathrm{GPa}$ [28]). The Nitinol transducer end-caps are fabricated with shape-setting before an annealing heat treatment was administered to adjust the transformation temperatures $[15,16]$. The composition of the superelastic Nitinol is $55.99 \mathrm{wt} \%$ nickel with a titanium balance. Small amounts of other elements such as copper, manganese, molybdenum, niobium, tungsten, silicon, chromium, cobalt and iron are also included for regulation of the transformation temperatures.

Assembled cymbal transducers are shown in Fig. 1. The binary and Nitinol transducers both contain a Navy Type I piezoceramic disc, Sonox ${ }^{\circledR}$ P4 (CeramTec GmbH), which is 
poled in the thickness direction. The piezoceramic disc, of 1.00 $\mathrm{mm}$ thickness and $12.70 \mathrm{~mm}$ diameter, possesses thickness and radial mode frequencies of $2 \mathrm{MHz}$ and $174 \mathrm{kHz}$ respectively. An insulating epoxy resin, chosen for its higher strength compared to conductive types [29], forms the mechanical coupling. A layer of Eccobond ${ }^{\circledR}$ (Ellsworth Adhesives Ltd) epoxy resin, at a mixture ratio of three parts $45 \mathrm{LV}$ resin to one part $15 \mathrm{LV}$ catalyst hardener, is deposited between the end-caps and the piezoceramic disc and is subsequently cured for 24 hours under an applied pressure. For each transducer, very small solder spots were deposited around the edge of the piezoelectric ceramic on each side, just enough to allow interaction with the transducer end-caps. Post-cure, electrode wires were attached to each end-cap using a conductive epoxy resin (Chemtronics CW2400) instead of soldering, to avoid the risk of thermal damage to the transducers, and in particular to the Nitinol end-caps.

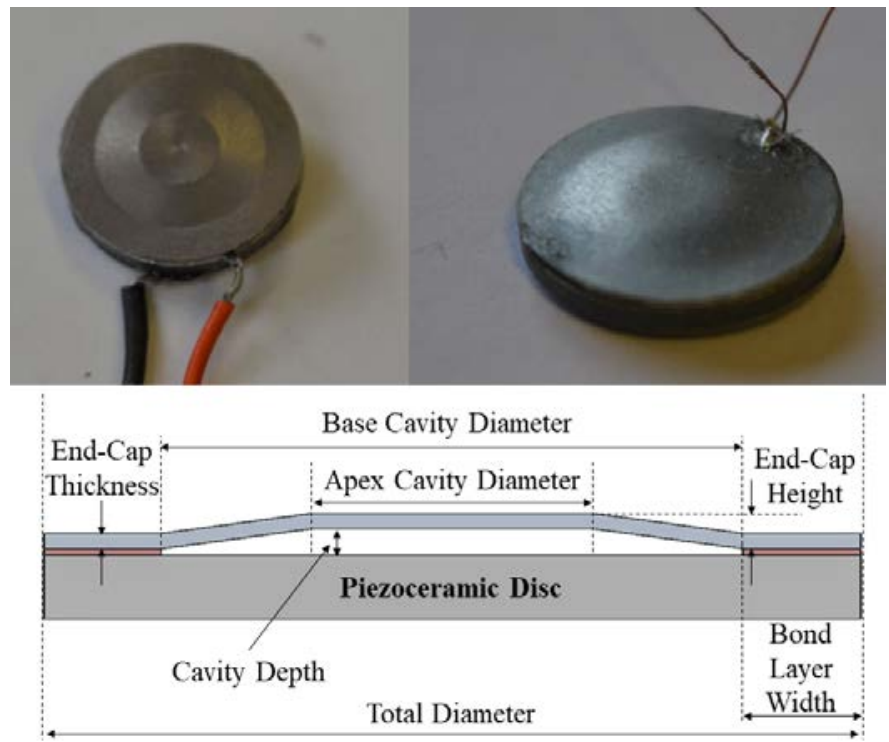

Fig. 1. The binary (top left) and Nitinol (top right) transducers. A side-view of the configuration of the end-cap and bond layer (bottom).

\section{Characterisation Results}

\section{A. Resonance Frequency Identification}

The impedance-frequency response is shown in Fig. 2, identifying the resonance frequencies. The results demonstrate the tuneable resonance capability of the transducers. For the binary transducer, Fig. 2(a), two resonance frequencies, indicating two distinct modes of vibration, are exhibited in the response spectrum, one at $25850 \mathrm{~Hz}$, and a second at $30475 \mathrm{~Hz}$. The vibration modes associated with the two frequencies are principally the same, but with one dominated by motion of the silver steel end-cap and the other dominated by motion of the titanium end-cap. The cap motion dominating the mode is then identified from experimental modal analysis.

Fig. 2(b) shows how the resonance frequencies of the Nitinol transducer increase in response to the temperature rise, where the Nitinol end-caps have undergone a phase transformation and thereby a change in the physical properties. Three resonance frequencies are detected at room temperature, at $19525 \mathrm{~Hz}, 22175 \mathrm{~Hz}$, and $40750 \mathrm{~Hz}$. As temperature was raised, the measured frequencies of the Nitinol transducer gradually increased until approximately $45^{\circ} \mathrm{C}$. Thereafter, frequency did not noticeably change with increasing temperature. The resonance frequencies increased to $23050 \mathrm{~Hz}, 25450 \mathrm{~Hz}$, and $41175 \mathrm{~Hz}$ respectively. The tuneability of the resonance response of the Nitinol transducer has hence been demonstrated, where the resonance frequencies can be increased through a rise from room temperature of approximately $30^{\circ} \mathrm{C}$.

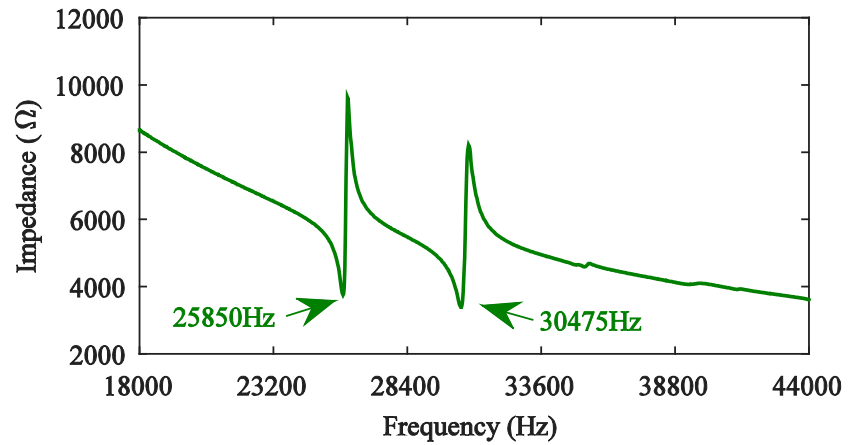

(a)

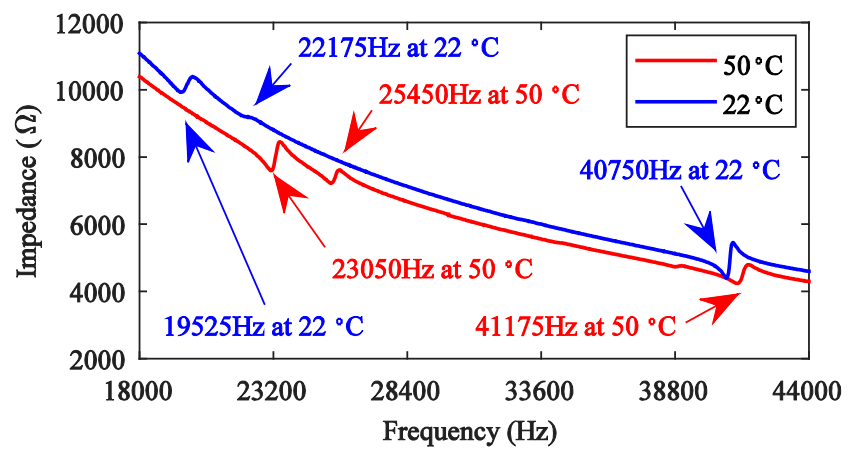

(b)

Fig. 2. Impedance-frequency response of (a) the binary transducer, and (b) the Nitinol transducer, measured at temperatures of $22^{\circ} \mathrm{C}$ and $50^{\circ} \mathrm{C}$, to demonstrate the Nitinol phase transformation effect.

The impedance of the Nitinol transducer decreased as the temperature was raised, as shown in Fig. 2(b). This behavior can be attributed to the pyroelectric effect, which can occur in some piezoelectric-based devices [30]. As the transducer is heated, a voltage is produced within the piezoelectric material, which can contribute to a decrease in the measured electrical impedance.

\section{B. Mode Shape Identification}

Mode Shape Prediction. The fundamental operating mode of a single-resonance cymbal transducer is a symmetric out-ofphase axial motion of the end-caps. When there is asymmetry between the end-caps or epoxy resin bond layers, two axial modes of vibration exist, the symmetric mode involving out-ofphase motion of the end-caps, and the asymmetric mode involving in-phase motion. FEA is a useful method for predicting the mode shapes of a cymbal transducer, for differentiating between modes of vibration, and for comparing 
with characterisation through EMA which allows for validation of FEA design. The fundamental mode shapes of a cymbal transducer are displayed in Fig. 3, from the FEA of a cymbal transducer, where the symmetric mode is shown in Fig. 3(a), and the asymmetric mode is exhibited in Fig. 3(b).

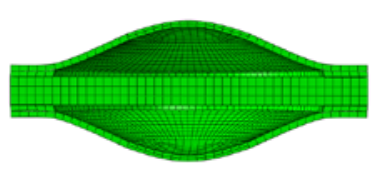

(a)

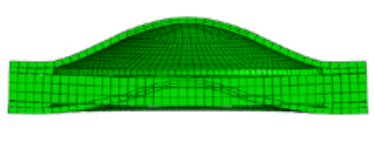

(b)
Fig. 3. The fundamental modes of a cymbal transducer, showing (a) the symmetric mode, and (b) the asymmetric mode.

In general, the order in which these mode shapes appear in a frequency response spectrum depends on the end-cap dimensions [4]. However, even with a fixed set of end-cap dimensions, the fabrication process can change this order, so that for some configurations the symmetric mode will appear at a lower frequency than the asymmetrical mode and for others will appear at a higher frequency. Furthermore, where there are differences between end-cap dimensions or materials, such as for the binary transducer configuration, two symmetric modes can occur, associated with dominant vibration motion of each end-cap.

TABLE II

MODE SHAPE ORDER FROM SIMULATION

\begin{tabular}{|c|c|c|}
\hline \multirow[b]{2}{*}{ Bond Layer Width (mm) } & \multicolumn{2}{|c|}{ Mode Shape } \\
\hline & $\begin{array}{l}\text { Mode } 1 \text { (Lower } \\
\text { Frequency) }\end{array}$ & $\begin{array}{l}\text { Mode } 2 \text { (Higher } \\
\text { Frequency) }\end{array}$ \\
\hline $\begin{array}{l}\mathrm{W}_{\text {END-CAP ST }}=2.25 \\
\mathrm{~W}_{\text {END-CAP TI }}=1.55\end{array}$ & Asymmetric & Symmetric \\
\hline $\begin{array}{l}\mathrm{W}_{\text {END-CAP ST }}=1.55 \\
\mathrm{~W}_{\text {END-CAP TI }}=2.25\end{array}$ & Asymmetric & Symmetric \\
\hline $\begin{aligned} \mathrm{W}_{\text {END-CAP ST }} & =1.25 \\
\mathrm{~W}_{\text {END-CAP TI }} & =1.55\end{aligned}$ & Symmetric & Symmetric \\
\hline $\begin{array}{l}\mathrm{W}_{\text {END-CAP ST }}=1.55 \\
\mathrm{~W}_{\text {END-CAP TI }}=1.25\end{array}$ & Asymmetric & Symmetric \\
\hline $\begin{aligned} \mathrm{W}_{\text {END-CAP ST }} & =1.05 \\
\mathrm{~W}_{\text {END-CAP TI }} & =1.55\end{aligned}$ & Symmetric & Symmetric \\
\hline $\begin{array}{l}\mathrm{W}_{\text {END-CAP ST }}=1.55 \\
\mathrm{~W}_{\text {END-CAP TI }}=1.05\end{array}$ & Asymmetric & Symmetric \\
\hline $\begin{aligned} \mathrm{W}_{\text {END-CAP ST }} & =1.25 \\
\mathrm{~W}_{\text {END-CAP TI }} & =1.05\end{aligned}$ & Symmetric & Asymmetric \\
\hline $\begin{array}{l}\mathrm{W}_{\text {END-CAP ST }}=1.05 \\
\mathrm{~W}_{\text {END-CAP TI }}=1.25\end{array}$ & Symmetric & Symmetric \\
\hline
\end{tabular}

Using FEA and the data in Table I, the order in the frequency domain in which the modes appear (i.e. lower or higher frequency) was calculated for different bond layer sizes in the binary transducer. In each model, a silver-steel and a titanium end-cap were included, and set to the dimensions as shown in Table I. In a cymbal transducer, the bond layers are created through deposition of epoxy resin in a ring formation around the end-cap flanges. For the simulation of different bond layer sizes, the width, $\mathrm{W}$, of the bond layer rings was adjusted and the bond layer radii were selected based on the fabricated binary transducer, but with minor adjustments. The outer radius of all bond layer rings was fixed at $6.35 \mathrm{~mm}$, the radius of each end- cap, and the thickness of all bond layers was $0.2 \mathrm{~mm}$. The results are shown in Table II.

It is clear that the fundamental modes are strongly affected by the widths of the epoxy resin bond layers. From the information presented in Table II, there is evidently a dependency both on the geometry of the transducer components and the relative dimensions of the simulated bond layers. Without sufficient control of the deposited epoxy layers in fabricated cymbal transducers, it is very difficult to rely on the modal frequency predictions of finite element models. Not only is bond layer size important, in terms of which mode shape is associated with which of the two frequencies, but so is the bond layer geometry that is associated with different end-cap geometries.

Mode Shape Measurements. The mode shapes and associated resonance frequencies measured from EMA for the binary transducer is shown in Fig. 4. The two fundamental modes of vibration can both exist as symmetric modes, often referred to as $(0,1)$ modes in the literature [13], and these modes are associated with a dominant motion of each end-cap [13]. This is illustrated in the EMA and FEA results in Fig. 4.

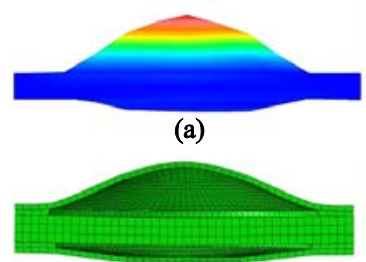

(c)

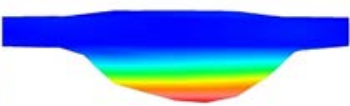

(b)

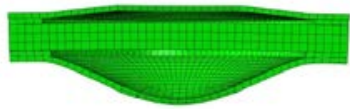

(d)
Fig. 4. Mode shapes of the binary transducer, showing (a) the Sym $\mathrm{ST}$ mode at $26056 \mathrm{~Hz}$ from EMA, (b) the $\mathrm{Sym}_{\mathrm{TI}}$ mode at $30648 \mathrm{~Hz}$ from EMA, where cool colours represent low amplitude vibration, and warmer colours signify higher amplitude vibration, (c) the Sym sT $_{\text {mode at }} 25759 \mathrm{~Hz}$ from FEA, and (d) the $\mathrm{Sym}_{\mathrm{TI}}$ mode at $30571 \mathrm{~Hz}$ from FEA.

The symmetric mode associated with motion of the silver steel end-cap is at $26056 \mathrm{~Hz}$, termed the Sym $\mathrm{ST}_{\mathrm{T}}$ mode, and the asymmetric mode at $30648 \mathrm{~Hz}$ is associated with dominant motion of the titanium end-cap, referred to as the $\mathrm{Sym}_{\mathrm{TI}}$ mode. The dual-resonance capability of this transducer therefore relies on being able separately to take advantage of the actuation motion of each end-cap. The mode shapes and resonance frequencies calculated using FEA correlate closely with those measured using electrical impedance analysis, where the discrepancy in the Symst mode is $0.35 \%$, and $0.31 \%$ for the Sym $_{\text {TI }}$ mode. The differences between the resonance frequencies of the binary transducer measured using electrical impedance analysis with those found from EMA for the Sym ${ }_{\mathrm{ST}}$ mode and the $S_{\text {TI }}$ modes are $0.79 \%$ and $0.56 \%$ respectively. The discrepancies between results from the experimental techniques largely arise due to the difference in measurement resolution. For example, the resolution of the EMA over the 0$80000 \mathrm{~Hz}$ frequency range is $1.56 \mathrm{~Hz}$, whereas it is $25 \mathrm{~Hz}$ for electrical impedance analysis data over the $18000-44000 \mathrm{~Hz}$ range.

The mode shapes and resonance frequencies of the Nitinol transducer were measured using the same method, and the 
results are shown in Fig. 5. Referring for comparison to the results in Fig. 2(b), there was a double-resonance measured in the impedance-frequency spectrum at $22^{\circ} \mathrm{C}$, which indicated that both a symmetric and an asymmetric mode of vibration existed in close frequency proximity, but the mode at $22175 \mathrm{~Hz}$ was less distinguishable in the spectrum compared to the mode at $19525 \mathrm{~Hz}$.

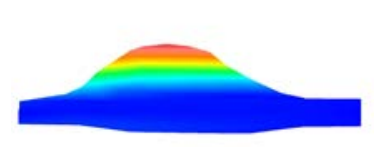

(a)

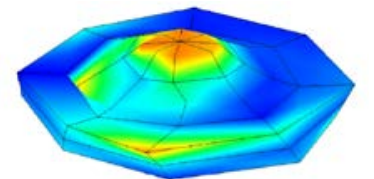

(b)

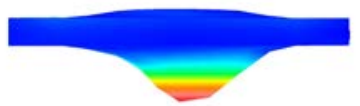

(c)

Fig. 5. Mode shapes of the Nitinol transducer, showing (a) the Asym ${ }_{N 11}$ at $19466 \mathrm{~Hz}$, (b) the $\mathrm{Sym}_{\mathrm{N} 11}$ at $21603 \mathrm{~Hz}$ and (c) the $\mathrm{Asym}_{\mathrm{NI} 2}$ mode at $40543 \mathrm{~Hz}$, measured at a temperature of $20^{\circ} \mathrm{C}$, where cool colours represent low amplitude vibration, and warmer colours signify higher amplitude vibration.

From the EMA measurements, a double-resonance was also measured, at $19466 \mathrm{~Hz}$ and $21603 \mathrm{~Hz}$. The mode of vibration measured at $19466 \mathrm{~Hz}$ was found to be the fundamental asymmetric mode, termed Asym ${ }_{\mathrm{NI} 1}$. However, two asymmetric modes were identified in the response, with the second, Asym $_{\mathrm{NI} 2}$, measured at $40543 \mathrm{~Hz}$, not being a fundamental asymmetric mode. This provides an explanation of why three modes are measured in the frequency response. The mode at $21603 \mathrm{~Hz}$ was found to be a symmetric mode, $\mathrm{Sym}_{\mathrm{N} 11}$, but exhibited a much lower response that the two asymmetric modes, Asym ${ }_{\mathrm{N} 11}$ and Asym $_{\mathrm{NI} 2}$. The differences in resonance frequencies measured using EMA and electrical impedance analysis were $0.30 \%, 2.58 \%$ and $0.51 \%$ for the Asym $_{\mathrm{NI} 1}$, Sym $_{\mathrm{NI} 1}$, and Asym ${ }_{\mathrm{N} 2}$ modes respectively.

The results show that identifying the mode shapes through EMA is very important in vibration characterisation of ultrasonic devices. In this case, EMA allowed fundamental modes to be distinguished from a higher order mode and demonstrated that, for some configurations, one of the two fundamental modes can be so dominant that the second fundamental may be very difficult to measure or observe in the frequency response. It is not possible to obtain this information from impedance-frequency measurements.

\section{Response of Transducers Driven at Resonance}

The binary transducer was excited in a frequency range through the resonance frequencies of the measured modes of vibration, at an excitation voltage amplitude of $2 \mathrm{~V}$. The displacement amplitude of the transducer in each mode of vibration is shown in Fig. 6 as a function of frequency, where the measurement for the displacement in the Sym $\mathrm{ST}_{\mathrm{ST}}$ mode is taken on the silver steel end-cap and for the $\mathrm{Sym}_{\mathrm{TI}}$ on the titanium end-cap.

The peak displacement amplitude of the Sym $\mathrm{ST}_{\mathrm{ST}}$ mode was measured as $1.52 \mu \mathrm{m}$ at $25996 \mathrm{~Hz}$, and the $\mathrm{Sym}_{\mathrm{TI}}$ mode was measured as $1.44 \mu \mathrm{m}$ at $30613 \mathrm{~Hz}$. The binary transducer exhibited these peak displacement amplitudes at resonance frequencies which correlate very closely with those of the Sym $_{\mathrm{ST}}$ mode and Sym $\mathrm{TI}_{\mathrm{TI}}$ mode measured using EMA $(0.23 \%$ and $0.11 \%$ respectively), electrical impedance analysis $(0.56 \%$ and $0.45 \%$ respectively) and FEA ( $0.91 \%$ and $0.14 \%$ respectively). The harmonic analysis method was conducted with a measurement resolution of $1 \mathrm{~Hz}$, thereby resulting in the minor discrepancies between the results of different experimental characterisation techniques.

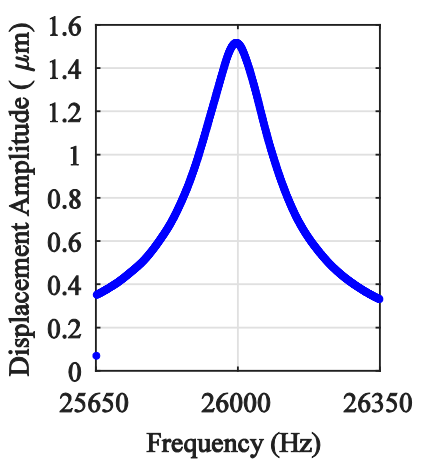

(a)

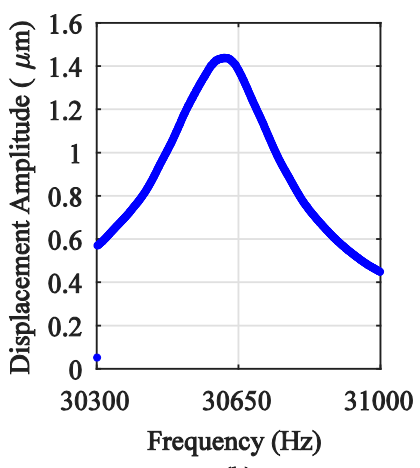

(b)
Fig. 6. Harmonic analysis of the binary transducer for a $2 \mathrm{~V}$ excitation voltage amplitude, showing (a) the Sym $\mathrm{ST}_{\mathrm{T}}$ mode, and (b) the Sym $\mathrm{SI}_{\mathrm{TI}}$ mode.

To investigate the influence of Nitinol end-cap superelasticity on the vibration response of the Nitinol transducer, the device was first driven at resonance in both Asym $_{\mathrm{NI} 1}$ and Asym $\mathrm{NI2}$ modes of vibration, with the Nitinol endcaps in the austenite phase, to determine the difference in the displacement amplitude response between the two modes. The mode of vibration exhibiting the higher displacement amplitude response was the Asym $\mathrm{NI2}_{\mathrm{I}}$ mode, measured to be $0.31 \mu \mathrm{m}$ at $41496 \mathrm{~Hz}$, compared to $0.23 \mu \mathrm{m}$ at $23545 \mathrm{~Hz}$ for the Asym ${ }_{\mathrm{NI}}$ mode. The transducer was hence operated in the Asym ${ }_{\mathrm{NI} 2}$ mode with the transducer heated to produce the austenite phase in the end-caps, and the results of the harmonic analysis for different excitation voltage amplitudes are shown in Fig. 7.

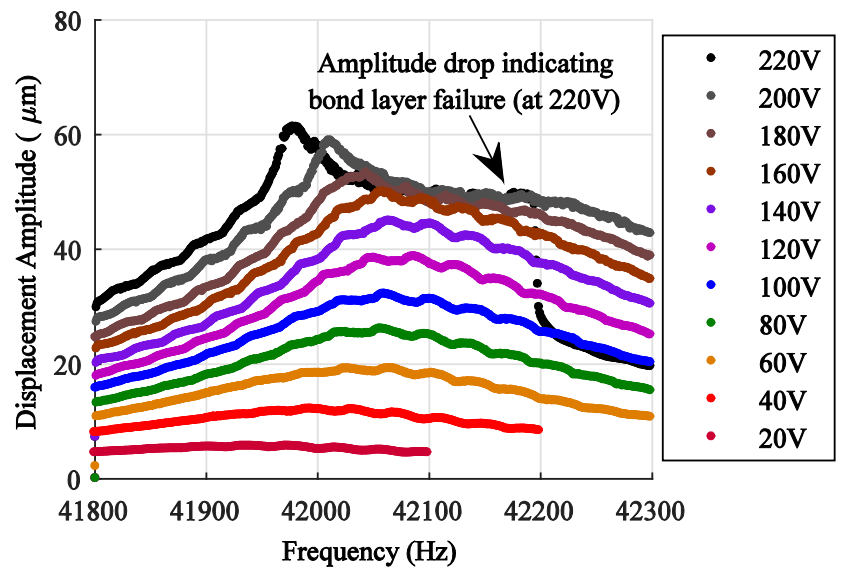

Fig. 7. Harmonic analysis of the Nitinol transducer operating in the Asym $\mathrm{N}_{\mathrm{N} 2}$ mode at $58^{\circ} \mathrm{C}$, for excitation voltage amplitude levels of $20-220 \mathrm{~V}$.

The displacement amplitude of the Nitinol transducer increased with excitation voltage, as shown in Fig. 7, reaching 
$60 \mu \mathrm{m}$ at $220 \mathrm{~V}$. At this voltage, a significant reduction in displacement amplitude was detected at $42200 \mathrm{~Hz}$. The mechanism of failure was most likely debonding in the epoxy layer, but the important observation from this experiment is that the amplitude-frequency response did not exhibit a noticeable characteristic difference to those amplitude-frequency measurements recorded at the lowest excitation levels. It is postulated therefore, that for this transducer the stress generated in the Nitinol end-caps is not sufficient to achieve a stressinduced martensitic transformation.

A significant observation from the harmonic analysis results of the Nitinol transducer is that the harmonic response is not stable, evidenced by modulations detected in the amplitudefrequency response. This behaviour has previously been identified in cymbal transducers $[15,16]$ and reported to be attributable to the thermal softening and degradation of the epoxy bond layers. Therefore, temperature is a variable that has a significant effect on the operation of cymbal transducers.

\section{Practical Application of the Transducers}

There is an opportunity to expand the number of applications for tuneable resonance transducers through the transducer design concepts presented here. Based on the two general approaches for transducer design that have been outlined, a tuneable resonance device for environments with variable temperature could exploit a form of the Nitinol transducer, and an application requiring a selectable operating frequency function could adopt the binary transducer. The advantage of the Nitinol cymbal is that it operates in a mode where the two end-caps have the same displacement at the two frequencies and so can more readily accommodate an attachment (such as a cutting tip). For the binary transducer, the higher displacement is on a different end-cap for the two frequencies so both endcaps would need to accommodate an attachment.

The Nitinol transducer has also shown promise for a second type of tuneable resonance transducer. The resonance frequencies can be controlled through a temperature change, and the transducer can be driven at resonance in the same mode at two different frequencies or in more than one mode of vibration. Furthermore, the superelasticity of the Nitinol endcaps appears to contribute no detectable influence on the vibration response of the transducer.

Although it is shown that the resonance frequencies of the Nitinol transducer can be controlled with temperature, there are complications relating to Nitinol that are not applicable to the binary transducer. First, the dissipation of heat after the phase transformation occurs at the higher temperatures is not instantaneous, and the transducer has to be cooled before an experiment can be repeated. This would be a serious limitation in a practical application, where a rapid transition between two resonance frequencies may be required. This supports previous concerns regarding some smart materials [31], but could be accommodated by incorporating a cooling system. Another complication is the manufacture of the phase-transforming SMA. In general, it is difficult to tune the desired transformation temperatures through the appropriate alloy composition, and cold working and heat treatment processes necessary for end-cap fabrication. However, these manufacturing challenges may be resolved through the improvement in the understanding of the mechanical properties and the static and dynamic behaviours of these materials.

\section{CONCLUSION}

The tuning of cymbal transducer resonance has been demonstrated through the application of two design and fabrication methods. A cymbal transducer with tuneable resonance capability can be produced using common cymbal transducer end-cap materials. As a novel alternative method, superelastic Nitinol SMA was incorporated into a cymbal transducer as the end-cap material. It was demonstrated that the transformational properties of superelastic Nitinol enable device resonance to be tuned in response to a temperature change, with no ostensible influence on transducer dynamic response characteristics resulting from the superelasticity of the Nitinol end-caps. However, further investigation is necessary to better understand the contributions from stress-induced martensite on the vibration response of a superelastic Nitinol transducer. With improvements to the transducer mechanical coupling, and advances in the understanding of the behaviour of Nitinol for ultrasonic devices, there is considerable scope for the development of tuneable resonance, power ultrasonic devices based on the cymbal transducer.

\section{ACKNOWLEDGMENT}

This research has been funded by the Engineering and Physical Sciences Research Council (EPSRC) grant EP/P505534/1.

\section{REFERENCES}

[1] C. Peters, D. Maurath, W. Schock, F. Mezger, and Y. Manoli, "A closedloop wide-range tunable mechanical resonator for energy harvesting systems," Journal of Micromechanics and Microengineering, vol. 19, no. 9, p. 094004, 2009.

[2] R. Feng, Y. Zhao, C. Zhu, and T.J. Mason, "Enhancement of ultrasonic cavitation yield by multi-frequency sonication," Ultrasonics Sonochemistry, vol. 9, no. 5, pp. 231-236, 2002.

[3] J.L. Overcash and J.F. Cuttino, "Design and experimental results of a tunable vibration turning device operating at ultrasonic frequencies," Precision Engineering, vol. 33, no. 2, pp. 127-134, 2009.

[4] R.J. Meyer Jr., W.J. Hughes, T.C. Montgomery, D.C. Markley, and R.E. Newnham, "Design of and fabrication improvements to the cymbal transducer aided by finite element analysis," Journal of Electroceramics, vol. 8, no. 2, pp. 163-174, 2002.

[5] R.E. Newnham, J. Zhang, and R. Meyer, Jr., "Cymbal transducers: a review," Proceedings of the IEEE International Symposium on Applied Ferroelectrics, pp. 29-32, 2000.

[6] S. Lin, "An improved cymbal transducer with combined piezoelectric ceramic ring and metal ring,” Sensors and Actuators A: Physical, vol. 163, no. 1, pp. 266-276, 2010.

[7] F. Bejarano, A. Feeney, and M. Lucas, "Vibration characterisation of cymbal transducers for power ultrasonics applications," Journal of Physics: Conference Series, IOP Publishing, vol. 382, no. 1, p. 012063, 2012.

[8] F. Bejarano, A. Feeney, and M. Lucas, “A cymbal transducer for power ultrasonics applications,” Sensors and Actuators A: Physical, vol. 210, pp. 182-189, 2014.

[9] F. Bejarano, A. Feeney, R. Wallace, H. Simpson, and M. Lucas. "An ultrasonic orthopaedic surgical device based on a cymbal transducer," Ultrasonics, vol. 72, pp. 24-33, 2016.

[10] P. Ochoa, J.L. Pons, M. Villegas, and J.F. Fernandez, "Effect of bonding layer on the electromechanical response of the cymbal metal-ceramic 
piezocomposite," Journal of the European Ceramic Society, vol. 27, no. 23, pp. 1143-1149, 2007.

[11] P. Ochoa, J.L. Pons, M. Villegas, and J.F. Fernandez, "Advantages and limitations of cymbals for sensor and actuator applications," Sensors and Actuators A: Physical, vol. 132, no. 1, pp. 63-69, 2006.

[12] P. Ochoa, M. Villegas, J.L. Pons, P. Leidinger, and J.F. Fernández, "Tunability of cymbals as piezocomposite transducers," Journal of Electroceramics, vol. 14, no. 3, pp. 221-229, 2005.

[13] J.F. Tressler and R.E. Newnham, "Doubly resonant cymbal-type transducers," IEEE Transactions on Ultrasonics, Ferroelectrics and Frequency Control, vol. 44, pp. 1175-1177, 1997.

[14] R.J. Meyer, Jr. and R.E. Newnham, "Flextensional transducers with shape memory caps for tunable devices," Journal of Intelligent Material Systems and Structures, vol. 11, no. 3, pp. 199-205, 2000.

[15] A. Feeney and M. Lucas, "Smart cymbal transducers with nitinol end caps tunable to multiple operating frequencies,", IEEE Transactions on Ultrasonics, Ferroelectrics and Frequency Control, vol. 61, no. 10, pp. 1709-1719, 2014.

[16] A. Feeney and M. Lucas, "Differential scanning calorimetry of superelastic nitinol for tunable cymbal transducers," Journal of Intelligent Material Systems and Structures, vol. 27, no. 10, pp. 1376-1387, 2016.

[17] S.A. Thompson, "An overview of nickel-titanium alloys used in dentistry," International Endodontic Journal, vol. 33, no. 4, pp. 297-310, 2000.

[18] T.W. Duerig and A.R. Pelton, “Ti-Ni shape memory alloys” In: G. Welsch, R. Boyer and E.W. Collings (eds), Materials Properties Handbook: Titanium Alloys, Materials Park, OH: ASM International, pp. 1035-1048, 1994.

[19] J.A. Shaw, C.B. Churchill, and M.A. Iadicola, "Tips and tricks for characterizing shape memory alloy wire: part 1 - differential scanning calorimetry and basic phenomena," Experimental Techniques, vol. 32, no. 5, pp. 55-62, 2008.

[20] K.E. Perry and P.E. Labossiere, "Phase transformations in nitinol and challenges for numerical modeling," Medical Device Materials II, ASM International, pp. 131-134, 2004.

[21] M.N. Bach, Kabir, A. Goyal, R. Malliwal, S. Kachrilas, M.E.E. Howairis, J. Masood, N. Buchholz, and I. Junaid, "A self-expanding thermolabile nitinol stent as a minimally invasive treatment alternative for ureteral strictures in renal transplant patients,” Journal of Endourology, vol. 27, no. 12, pp. 1543-1545, 2013.

[22] Y. Liu and H. Xiang, "Apparent modulus of elasticity of near-equiatomic NiTi," Journal of Alloys and Compounds, vol. 270, no. 1, pp. 154-159, 1998.

[23] M. Drexel, G. Selvaduray, and A. Pelton, "The effects of cold work and heat treatment on the properties of nitinol wire," Medical Device Materials IV: Proceedings from the Materials \& Processes for Medical Devices Conference 2007, pp. 114-119, 2008.

[24] J. Olbricht, A. Yawny, J.L. Pelegrina, A. Dlouhy, and G. Eggeler, "On the stress induced formation of R-phase in ultra-fine-grained ni-rich NiTi shape memory alloys,” Metallurgical and Materials Transactions A, vol. 42, no. 9, pp. 2556-2574, 2011.

[25] D. Stöckel, "Nitinol - a material with unusual properties," Endovascular Update, no. 1, pp. 1-8, 1998.

[26] J.F. Tressler, W. Cao, K. Uchino, and R.E. Newnham, "Finite element analysis of the cymbal-type flextensional transducer," IEEE Transactions on Ultrasonics, Ferroelectrics and Frequency Control, vol. 45, pp. 13631369, September 1998.
[27] S. Arbós-Torrent, B. Ganapathisubramani, and R. Palacios, "Leading- and trailing-edge effects on the aeromechanics of membrane aerofoils," Journal of Fluids and Structures, vol. 38, pp. 107-126, 2013.

[28] M. Long and H.J. Rack, "Titanium alloys in total joint replacement - a materials science perspective,” Biomaterials, vol. 19, no. 18, pp. 16211639, 1998.

[29] J. Zhang, A.-C. Hladky-Hennion, W.J. Hughes, and R.E. Newnham, "Modeling and underwater characterization of cymbal transducers and arrays," IEEE Transactions on Ultrasonics, Ferroelectrics and Frequency Control, vol. 48, no. 2, pp. 560-568, 2001.

[30] F.G. Baptista, D.E. Budoya, V.A.D. de Almeida, and J.A.C. Ulson, “An experimental study on the effect of temperature on piezoelectric sensors for impedance-based structural health monitoring," Sensors, vol. 14, no. 1, pp. 1208-1227, 2014.

[31] J.M. Jani, M. Leary, A. Subic, and M.A. Gibson. "A review of shape memory alloy research, applications and opportunities," Materials \& Design, vol. 56, pp. 1078-1113, 2014.

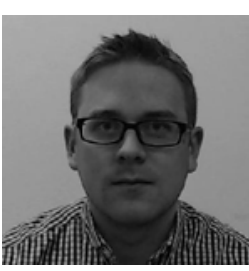

Andrew Feeney graduated with a M.Eng. degree in mechanical engineering from the University of Glasgow, followed by a Ph.D. degree from the same institution. He is currently Research Fellow at the University of Warwick. His research interests are devices for industrial ultrasonics and smart materials for ultrasound applications.

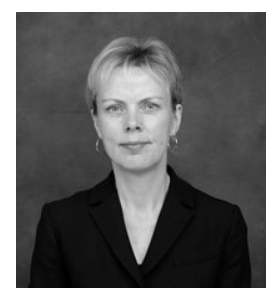

Margaret Lucas obtained a B.Sc.(Eng.) degree in mechanical engineering from the University of Aberdeen, followed by a $\mathrm{Ph} . \mathrm{D}$. degree from Loughborough University. She was a lecturer in dynamics at Loughborough University before moving to the University of Glasgow in 1996, where she is currently Professor of Ultrasonics. Her research interests are in industrial and medical applications of power ultrasonics. 